\title{
Grammatische Terminologie in lateinischen Sprachbüchern
}

Wenn im Blick auf eine Vereinheitlichung grammatischer Terminologie ein eigenes Referat über lateinische Sprachbücher vorgesehen ist, dann ergibt sich das zunächst einfach aus der Tatsache, daß Latein ein Teil des an den schulen etablierten sprachunterrichts ist. Doch stellen sich dabei sicher auch noch andere Uberlegungen ein. Lange zeit waren die grammatische Terminologie und auch die Beschreibungsverfahren im Deutschunterricht und in modernen Fremdsprachen von der lateinischen Grammatik her geprägt. Inzwischen setzten sich neue, den einzelnen Sprachen angemessenere Beschreibungsweisen durch, neue Grammatiktheorien wurden entwickelt. Verständicherweise war damit die Neigung verbunden, auch neue Termini einzuführen. Der Lateinunterricht kam dadurch in eine schwierige situation. Einerseits sah man (vom standpunkt des Lateinlehreres aus) nicht ein, das man an den Termini, die sich im Umgang mit der lateinischen Grammatik entwickelt hatten, nicht festhalten sollte. Andererseits forderten neue Beschreibungsweisen auch zur Uberprüfung der traditionellen lateinischen Grammatik heraus. Dies wiederum mubte auch zu einer kritischen Uberprüfung der Termini führen. Eine gewisse Unsicherheit war die Folge.

Von der Praxis des sprachunterrichts in den Schulen ging eine weitere Verunsicherung aus. In dem Entwurf einer 'Basisliste grammatischer Fachausdrücke', den die Landesstelle für Erziehung und Unterricht in Stuttgart 1980 vorlegte, heibt es S. 7: "Dem Fach Deutsch obliegt es natürlicherweise, das Fundament für die schulgrammatische Arbeit zu legen und Anstöße und Ansätze zu einer 'Verstehensgrammatik' zu geben, die über die Einzelsprachen hinweg die sprache ins Blickfeld der Betrachtung rückt." Diese Forderung ist durchaus begründet. Aber die Wirklichkeit sah oft anders aus. Der Deutschunterricht erfüllte diese Funktion vielfach nicht mehr oder er gebrauchte unterschiedliche Termini. Der Lateinunterricht war davon in besonderer Weise betroffen. Er 
ist durch den primär reflektierenden Umgang mit der sprache auf die Entwicklung einer brauchbaren grammatischen Terminologie angewiesen, die nach Möglichkeit nicht nur auf das Lateinische anwendbar ist. AuBerdem ist nach wie vor eine der wichtigsten Tätigkeiten des Lateinunterrichts das Ubersetzen. Das schliebt eine ständige kontrastive Betrachtung mindestens lateinischer und deutscher Ausdrucksweisen ein. Wie hilfreich dabei eine weithin einheitliche grammatische Terminologie sein kann, ist ohne weiteres einzusehen.

Der Lateinunterricht hat auf Grund solcher Uberlegungen ein vitales Interesse an einer weitgehenden Vereinheitlichung grammatischer Termini im sprachunterricht. Daß dabei viele in der lateinischen Grammatik gebräuchliche Termini eine Rolle spielen, ist nicht zeichen irgendeines Vorranges des Lateinischen. Ebensowenig wird es darum gehen, Beschreibungsverfahren der lateinischen Grammatik auch dort anzuwenden, wo sie unangemessen sind. vielmehr bieten die tradierten Termini die Möglichkeit, unbefangener Erscheinungen $z u$ benennen, ohne sich mit den Benennungen schon in der Bestimmung der Erscheinungen festzulegen. der bereits zitierte Entwurf einer Basisliste grammatischer Fachausdrücke bemerkt s. 4 richtig: "Je weniger die grammatischen Termini kraft ihrere verblaßten Grundbedeutung an erschließbarem, ursprünglichem Wortsinn preisgeben, umso geeigneter erscheinen sie als 'Etiketten' für abstrakte Funktionen, die sich dem Schüler vielleicht erst im Laufe mehrerer Jahre voll erschlieben."

In den vergangenen 10-15 Jahren haben verschiedene Einflüsse neuerer Linguistik auf den Umgang mit der Grammatik in der schule eingewirkt. An erster stelle zu nennen ist die Valenz-bzw. Dependenzgrammatik. Diesem Grammatikmodell gegenüber hatte man die geringsten Vorbehalte. ${ }^{1}$ schwierigkeiten bereitete dagegen die Berücksichtigung transformationeller Aspekte. Textlinguistische Gesichtspunkte, die für die Texterschließung und für das Textverstehen hilfreich schienen, wurden weitgehend akzeptiert. 
Im ganzen kann man sagen, daß die Neigung der Lateinlehrer, sich auf neue Uberlegungen zur Grammatik einzulassen, nicht sehr groB ist. In den sprachbüchern herrschen traditionelle Beschreibungsverfahren vor, die durch neue Aspekte ergänzt werden. Vermittelt wird im wesentlichen die Morphologie in Verbindung mit einer Satzgliedgrammatik. Dabei werden Elemente der Valenzgrammatik (z.B. in Fontes, cursus Latinus, Nota) oder auch transformationelle Beziehungen berücksichtigt (besonders in contextus, z.T. auch in Fontes, 1 itterae, Nota, redde rationem). 2

Lehnt sich ein Buch an die Valenzgrammatik an, dann erscheint neben den traditionellen Benennungen der Satzglieder die Unterscheidung zwischen notwendigen Satzgliedern und freien zusätzen (so in Fontes), oder es wird zwischen Ergänzungen und freien Angaben unterschieden (Nota). Cursus Latinus führt die Unterscheidung von sinn-notwendigen Ergänzungen und verdeutlichenden Ergänzungen bzw. von adverbialen freien Angaben ein.

Das Vorgehen der Sprachbücher bietet ein einfaches Musterbeispiel für die probleme, die mit der Einführung neuer Beschreibungsverfahren und der ihnen zugeordneten Termini verbunden sind. Das Einbeziehen neuer syntaktischer Aspekte scheint geradezu zwangsläufig zu unterschiedlichen Termini zu führen. Dabei zeigen sich sofort auch Fragwürdigkeiten. Die rein syntaktischen Bestimmungen der valenzgrammatik bekommen im Cursus Latinus eine inhaltliche Tönung: Von $s$ i n n-notwendigen Ergänzungen, $v$ e rd e $u t l i c h$ e $n$ d e $n$ Ergänzungen ist die Rede. Das suggeriert die falsche Vorstellung, als seien die sinn-notwendigen Ergänzungen die eigentlich wichtigen, die verdeutlichenden Ergänzungen dagegen weniger wichtig.

An die Transformationsgrammatik wird angeknüpft, indem Erscheinungen wie Prädikativum, aci, ablativus absolutus, -nd-Formen, Gliedsätze durch Einbettung bzw. Transformation zugrunde liegender sätze erklärt werden. Am ausgeprägtesten ist diese Möglichkeit in Contextus genutzt. Dort werden die erwähnten Erscheinungen durchweg durch transformationelle Beziehungen erklärt. 
Einbettung bzw. Transformation sind ausdrücklich erörterte Verfahren. Der Satz, in den ein anderer Satz als Gliedsatz eingebettet wird, wird in contextus Matrixsatz genannt. Es hat sich aber gezeigt, daß die Lateinlehrer diesen Terminus nur ungern aufgreifen, weil er sonst in der schule kaum gebraucht wird. Man bleibt lieber bei den Bezeichnungen 'Hauptsatz' oder 'Ubergeordneter satz'.

In neueren lateinischen Sprachbüchern gibt es an einigen stellen auch Bemühungen um Verbesserungen der tradierten Terminologie. So führt Fontes Partizip I (Partizip Präsens (Aktiv)) und partizip II (Partizip Perfekt (Passiv)) ein (vgl. Fontes S. 67; 79; vgl. auch S. 151). Die Termini Partizip Präsens und Partizip perfekt sind mibverständich, da die Partizipien durch Tempusbezeichnungen unterschieden werden, aber keine Tempusbedeutung haben. Man könnte darauf verweisen, dab das Part. Präs. (Akt.) vom Präsensstamm gebildet ist, aber einen Perfekt-Passiv-stamm des Verbs gibt es nicht. Perf., Plusquamperf. und Fut. II Pass. werden bekanntlich durch zusammengesetzte Formen gebildet (Part. Perf. Pass. + Formen von essel. Da die Partizipien zeitverhältnisse ausdrücken, könnte man das partizip der Gleichzeitigkeit (Part. Präs.) und das der Vorzeitigkeit (Part. Perf.) unterscheiden.

Nun gibt es aber Fälle, in denen das Part. Perf. offensichtlich keine vorzeitigkeit ausdrückt. ${ }^{3}$ Auberdem müBten die Bezeichnungen 'Partizip der Gleichzeitigkeit' und 'Partizip der Vorzeitigkeit' im Unterricht dazu führen, daß mit dem ersten Vorkommen der Partizipien sogleich die Zeitverhältnisse mit erörtert würden. Das ist sicher nicht unmöglich, aber mancher wird das gern in einem $z$ weiten schritt tun. Allen Schwierigkeiten geht man aus dem Weg, wenn man einfach Partizip I (Part. Präs. Akt.) und Partizip II (Part. Perf. Pass.) unterscheidet und weitere Differenzierungen einbringt, wenn es zweckmäBig scheint. Man könnte dann das Part. Fut. Akt. als Partizip III bezeichnen. 
Diese Erörterung zeigt wichtige Aspekte für eine brauchbare grammatische Terminologie: Die Termini sollen dem Sprachgebrauch angemessen und offen sein für die nötigen Differenzierungen. Das Beispiel der Partizipien verweist noch auf einen anderen Gesichtspunkt: Man muß auf Bezüge zum weiteren sprachsystem achten. So müssen die Termini für die Partizipien im Lateinischen in Verbindung mit den Bezeichnungen für die Infinitive gesehen werden. Denn auch die Infinitive drücken Zeitverhältnisse, nicht Tempora aus, und zwar Inf. Präs. (Akt. und Pass.) Gleichzeitigkeit, Inf. Perf. (Akt. und Pass.) vorzeitigkeit (mit Ausnahmen ${ }^{4}$ ), Inf. Fut. (Akt. und Pass.) Nachzeitigkeit. Wenn man folgerichtig vorgeht, mübte man von Inf. I, Inf. II und Inf. III sprechen. Das wäre zwar konsequent, aber ich vermute, daß diese wiederholte Unterscheidung nur durch zahlen für den Sprachunterricht $z u$ wenig deutlich ist. Nach dieser Uberlegung müBte es am ehesten auf Benennung nach den zeitverhältnissen hinauslaufen, Man müBte dabei die geringfügigen Inkonsequenzen beim part. Perf. und beim Inf. Perf. in Kauf nehmen.

Das Verzeichnis der Kultusministerkonferenz läßt sich übrigens beim Infinitiv auf das problem nicht ein, nennt nur den Terminus Infinitiv, differenziert aber zwischen partizip I und Partizip II. Offensichtlich handelt es sich bei dem dargelegten Problem der Unterscheidung zwischen den Infinitiven um ein spezielles Problem des Lateinischen, so daB mit Recht in die Basisliste nur der Terminus Infinitiv aufgenommen wird. Immerhin wird aber erkennbar, welche Folgen es für das Lateinische haben kann, wenn die partizipien mit partizip I und partizip I unterschieden werden. Das soll nicht ein Argument gegen die vorgeschlagene Basisliste grammatischer Fachausdrücke sein, aber sie wird für jede sprache vergleichbare Fragen aufwerfen.

Noch eine andere stelle der Liste ist für das Lateinische besonders beachtenswert. In der Liste erscheint die Unterscheidung 'Konjunktiv I' und 'Konjunktiv II'. Von den neueren lateinischen Sprachbüchern entsprechen einige mit gewissen Abweichungen dieser Terminologie, andere bleiben bei Konj. Präs.,Imperf.,Perf., 
Plusquamperf. Contextus verwendet für Konj. Präs. und für Konj. Imperf. Akt. und Pass. die Bezeichnungen 'Konjunktiv I Präsensstamm' bzw. 'Konjunktiv II Präsensstamm', für Konj. Perf. und Konj. Plusquamperf. Akt. 'Konjunktiv I Perfektstamm' bzw. 'Konjunktiv II Perfektstamm', mus dann allerdings im Passiv, da es ja keinen Perfekt-Passiv-stamm gibt, 'Konjunktiv I Perf.-Pass.Reihe' und 'Konjunktiv II Perf.-Pass.-Reihe' unterscheiden. Nota differenziert durch fortlaufende zahlen: Konjunktiv I und Konjunktiv II für konj. Präs. und Imperf., dann Konjunktiv III und Konjunktiv IV für Konj. Perf. und Plusquamperf.

Ohne zweifel sind solche Bezeichnungen sinnvoller als die üblichen. Es ist z.B. eigentlich unsinnig, von einem Konj. Imperf. zu reden, da dieser Konjunktiv im Lateinischen keinerlei Beziehung - weder formal noch syntaktisch - zum Imperfekt und bei bestimmten Gebrauchsweisen (Irrealis der Gegenwart, unerfüllbarer Wunsch der Gegenwart) nicht einmal eine Beziehung zur Vergangenheit hat. Die Konjunktive drücken auberdem häufig nur zeitverhältnisse aus (vgl. consecutio temporum ${ }^{5}$ ). Der sogenannte Konj. Plusquamperf. z.B. ist dann meist nicht mit einem plusquamperfekt $z u$ übersetzen. Die tradierten Bezeichnungen stellen wirklich nur schematische Entsprechungen im system her und sind für das Verstehen von Sprache in Texten nicht hilfreich. Die unverfängliche Unterscheidung zwischen Konjunktiv I und Konjunktiv II verleitet dagegen nicht zu einem falschen verständnis und behindert so nicht Differenzierungen, die der jeweilige zusammenhang erfordert.

Kurz sei noch erwähnt, daß es in neueren lateinischen sprachbüchern im Bereich der syntax auch terminologische Ergänzungen gibt, die auf präzisierungen der sprachbeschreibung beruhen. Hier ist die Einführung des präpositionalen objekts und des Ablativ-objekts zu nennen.

Von besonderer Bedeutung ist der Gebrauch von Termini, die im Anschluß an Einsichten der Textlinguistik der Texterschließung und dem Verstehen der Textkonstitution dienen. Folgende Termini erscheinen in neueren Sprachbüchern ${ }^{6}$ : 
Repetition / wiederholung, Umschreibung / Paraphrase, wörter mit gemeinsamen inhaltlichen Merkmalen, Gegensatz (inhaltlich), Proformen,

Rückverweis (anaphorisch), Vorverweis (kataphorisch), Konnektor als satzverknüpfendes Wort, Thema - Rhema.

Es wäre sicher nützlich, wenn Termini zur TexterschlieBung und zum Textverstehen in ein Verzeichnis grundlegender grammatischer Fachausdrücke aufgenommen würden. Aus der Sicht des Lateinunterrichts scheinen mir mindestens folgende Termini erforderlich:

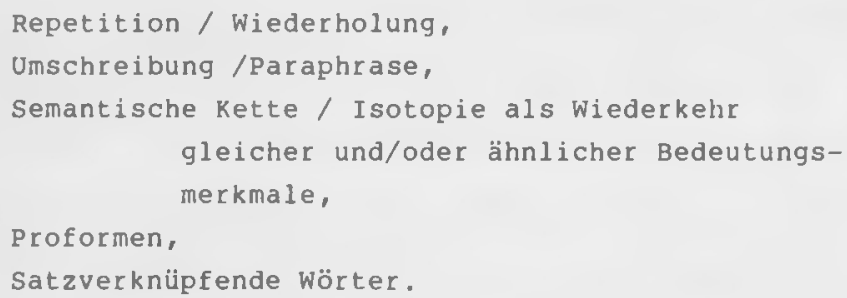

Zum SchluB möchte ich noch ein paar Bemerkungen zum "Verzeichnis grundlegender grammatischer Fachausdrücke" der Kultusministerkonferenz machen. Ich glaube, für den Lateinunterricht ist diese Liste brauchbar. Aber einige Ergänzungen über die erwähnten Termini zur Textebene hinaus wären sicher nicht nur für den Lateinunterricht nützlich. Der Begriff 'Kongruenz' kann vielleicht vom Standpunkt anderer Sprachen aus für die Basisliste entbehrt werden, aber das dürtte kaum für 'Aspekt' und 'Aktionsart' gelten. Im Bereich der syntax sollte man doch 'Funktion', 'Füllung von Satzgliedpositionen', die Erscheinung der 'Wortgruppe' berücksichtigen. Wichtig scheint mir auch ein Terminus wie 'Satzwertiges Satzglied', da er ein beachtliches Feld von Erscheinungen abdeckt. Auf die in sich widersprüchlichen Termini 'Partizipialsatz' und 'Infinitivsatz' würde ich gern verzichten. Das Prädi- 
kativum ${ }^{7}$ ist für den Lateinunterricht unentbehrlich, ob das auch für andere sprachen gilt, die in der schule gelehrt werden, müssen die vertreter dieser sprachen beurteilen.

Es scheint mir nicht so wichtig, daB man sich über möglichst viele Termini einer Basisliste einigt. Entscheidend ist, daB überhaupt ein solches Verzeichnis zustande kommt. Um manche Termini wird man immerzu streiten können. Das hilft den schülern wenig, für die es aber von großem Nutzen wäre, wenn der Unterricht in verschiedenen sprachen in einer Reihe von Termini eine einheitliche Grundlage hätte. Es geht dabei ja nicht nur um etwas ÄuBerliches. Vielmehr wird hier so etwas wie ein durchgängig brauchbarer orientierungsrahmen für die sprachen greifbar, die in der schule gelehrt werden. Die schüler werden sich im Umgang mit den sprachen besser zurechtfinden und sich in fachspezifische Modifikationen und Erweiterungen besser einarbeiten können. Es wäre töricht, bei diesem Unternehmen nicht tradierte Termini zu nutzen, wenn sie brauchbar sind. Das schliebt nicht aus, daß man dort, wo es zweckmäßig scheint, auch neue Fachausdrücke einführt. Aber das berechtigte Bemühen um bessere und den einzelnen Sprachen angemessenere Beschreibungsverfahren muB nicht notwendig zu völlig unterschiedlichen Terminologien führen. Für die Praxis des sprachunterrichts ist es jedenfalls hilfreich, daß man ebenso die gemeinsame Grundlage und das Miteinander im Umgang mit sprache beachtet wie auch Besonderheiten gerecht wird. 


\section{Anmerkungen}

1 Der Anschluß an die traditionelle Grammatik ist hier leicht herzustellen. Sicher empfanden viele auch eine Nähe zur sogenannten Konstruktionsmethode, bei der man ja vom prädikat aus die weiteren satzglieder zu erschließen versucht.

2 Folgende lateinische Lehrbijcher werden erwähnt:

redde rationem (Lehrgang für Latein als 1. und als 2. Fremdsprache)

Arbeitsbuch 1 von J. Malms

Arbeitsbuch 2 von W. Emrich und J. Malms

Arbeitsbuch 3 von G. Bloch, W. Emrich, J. Malms

Klett-Verlag, stuttgart.

Nota (fïr Latein als 3. Fremdsprache) von M. Fuhrmann, E. Hermes, H. Steinthal, N. Wilsing

Klett-Verlag, stuttgart.

Contextus von D. Gaul, w. Heilmann, W. Höhn, U. Pürzer

Verlag Moritz Diesterweg, Frankfurt am Main.

Fontes von H.-J. Glücklich, H. Holtermann, W. Zapfe

Verlag Vandenhoeck und Ruprecht, Göttingen.

Cursus Latinus, Grammatische Beihefte von F. Maier, R. Bauer, H. Grosser Buchners Verlag/Lindauer Verlag/Oldenbourg Verlag, Bamberg/München.

litterae (für spätbeginnendes Latein) von R. Nickel

Buchners Verlag/Lindauer Verlag/Oldenbourg Verlag, Bamberg/München.

Bornemann, Lateinisches Unterrichtswerk, Ausgabe C, neu bearbeitet von $\mathbf{E}$. Gebhardt, P. Kroh, K.W. Reinhardt

Hirschgraben-Verlag, Frankfurt am Main.

$3 \mathrm{Vgl}$. H. Menge, Repetition der lateinischen syntax und Stilistik, 14. Auf1., bes. von A. Thierfelder, Minchen 1965, $\$ 301$ und $\S 439$, Anm. 2c.

$4 \mathrm{vgl}$. Menge a.0. $\$ 417,1$.

5 Das führt bei redde rationem dazu, Konjunktiv I und II Gleichzeitigkeit von Konjunktiv I und II Vorzeitigkeit zu unterscheiden. Das ist eine fragwürdige Terminologie, da so nicht der ganze Gebrauch des Konjunktivs exfaßt wird.

6 vor allem in Contextus, Fontes, Bornemann: neue C-Ausgabe, weniger in Nota, litterae.

7 vgl. Sätze wie: Cicero consul ('als Konsul', 'als Cicero Konsul war') coniurationem detexit. Tarquinius Superbus a Romanis expulaus ('vertrieben'; 'nach seiner Vertreibung' u.a.) ad Etruscos confugit. 